Rabaska

Revue d'ethnologie de l'Amérique française

REID-MARCIL, EILEEN. On chantait "Charley-Man ». La construction des grands voiliers à Québec de 1763 à 1893. Québec, Les Éditions GID, 2000, 468 p. ISBN 2-922668-02-9

\title{
André Kirouac
}

Volume 2, 2004

URI : https://id.erudit.org/iderudit/201682ar

DOI : https://doi.org/10.7202/201682ar

Aller au sommaire du numéro

Éditeur(s)

Société québécoise d'ethnologie

ISSN

1703-7433 (imprimé)

1916-7350 (numérique)

Découvrir la revue

Citer ce compte rendu

Kirouac, A. (2004). Compte rendu de [REID-MARCIL, EILEEN. On chantait "Charley-Man ». La construction des grands voiliers à Québec de 1763 à 1893.

Québec, Les Éditions GID, 2000, 468 p. ISBN 2-922668-02-9]. Rabaska, 2 ,

255-257. https://doi.org/10.7202/201682ar d'utilisation que vous pouvez consulter en ligne.

https://apropos.erudit.org/fr/usagers/politique-dutilisation/ 
ReID-Marcil, EILEEN. On chantait " Charley-Man ». La construction des grands voiliers à Québec de 1763 à 1893. Québec, Les Éditions GID, 2000, 468 p. ISBN 2-922668-02-9.

Depuis près de 30 ans, Eileen Reid-Marcil consacre la majeure partie de ses recherches à la glorieuse époque des grands voiliers. On chantait "Charley-Man » est son second volume, le premier traitant de l'histoire des chantiers maritimes Davie.

La traduction française de cet ouvrage, originalement paru en anglais sous le titre « The Charley-Man, A History of Wooden Shipbuilding at Quebec 1763-1893", respecte assez fidèlement les propos de l'auteur. La structure des chapitres, ainsi que la majorité des textes et des illustrations reprennent ceux de la publication anglaise. Il faut cependant noter, comme l'auteur le précise, que quelques modifications ont été apportées ça et là, notamment lors des désignations toponymiques de la ville de Québec. Dans la version anglaise, madame Reid-Marcil précise qu'il est important de conserver les toponymes anglais qui reflètent mieux la réalité anglophone du $\mathrm{XIX}^{\mathrm{e}}$ siècle à Québec. Dans la version française, elle souligne plutôt que les toponymes de rues ont été traduits en français puisque le « livre s'adresse à un lectorat 
français [et] ensuite parce que la plupart des noms anglais n'ont plus cours aujourd'hui. » Cette précision linguistique dans les deux éditions apparaît comme contradictoire et il est permis de penser qu'elle aurait pu, sans nuire à la compréhension, conserver, dans l'édition française, les termes anglais en usage au $\mathrm{XIX}^{\mathrm{e}}$ siècle. L'auteur profite d'ailleurs de cette version française pour présenter bon nombre d'illustrations qui seront plus susceptibles d'intéresser les lecteurs francophones. Par exemple, une carte d'affaires d'un constructeur anglophone est remplacée par celle d'un constructeur francophone ou encore, un document $d$ 'archives en langue anglaise ne sera pas traduit mais plutôt substitué par un document français de même nature. Les subtilités de la traduction, outre pour les toponymes, ont à quelques reprises entraîné des erreurs techniques. Par exemple, en page 353 de l'édition anglaise " the 396 tons barquentine White Wings », devient en français « le trois-mâts goélette de 396 tonneaux », alors qu'on devrait lire le trois-mâts barque. L'erreur se retrouve ailleurs, notamment en page 319 de la version française où, dans la figure 209 , l'on décrit un trois-mâts goélette, qui est en fait, un trois-mâts barque.

Si le lecteur prend en considération la possibilité que quelques erreurs de traduction soient commises, 1'ouvrage de madame Reid-Marcil répond à sa volonté d'être accessible aux lecteurs de langue française. Les recherches effectuées, la diversité et la quantité de sources exploitées et les multiples références bibliographiques présentées attestent d'une réelle volonté d'offrir un portrait de ce que fut l'époque de la construction navale des années 1763 à 1893 à Québec. Il faut dire que le projet de l'auteur est colossal. Les neuf chapitres du volume, ses appendices et ses diverses listes de figures, cartes et plans contiennent une somme importante d'informations qui touchent la grande majorité des caractéristiques de la construction navale.

En introduction, madame Reid-Marcil précise qu'elle veut « se pencher sur l'ensemble du travail des personnes qui ont construit ces navires. » De même, elle souligne qu'elle peut aussi traiter de périodes historiques antérieures ou postérieures à celle qui l'intéresse si cela s'avère nécessaire à la bonne compréhension du texte. Elle explique qu'elle a choisi de présenter cette histoire de manière simple car elle souhaite rendre le volume accessible au grand public et aux descendants des constructeurs et des ouvriers des chantiers navals.

Dans le but d'atteindre les objectifs fixés, Eileen Reid-Marcil a donc choisi de diviser les chapitres de son livre en fonction des aspects touchant la construction des navires à Québec entre 1763 et 1893. Du commerce du bois à l'administration des chantiers et des principaux constructeurs aux différents corps de métiers, le lecteur pénètre dans un univers aujourd'hui disparu et qui est, pour plusieurs, totalement oublié, voire même inconnu. Les subdivi- 
sions de chacun des chapitres donnent un éclairage particulièrement complet des différentes thématiques abordées. Au chapitre cinq, l'auteur décrit les installations des chantiers. Presque tout y passe, autant la description des cales de construction que celle du bureau du comptable. Au chapitre huit, nous découvrons plus de seize métiers reliés à la construction navale. Encore là, chacun fait l'objet d'une description accompagnée d'illustrations aidant à la compréhension du lecteur. Enfin, en appendice, des listes, des illustrations ou des documents d'archives, des nomenclatures de noms et de caractéristiques de voiliers, de chantiers ou de constructeurs nous sont proposés.

Les lecteurs à la recherche de données factuelles et événementielles y trouveront donc une excellente source d'informations. En ce sens, l'ouvrage répond visiblement à l'un des objectifs de l'auteur : faire découvrir aux descendants des constructeurs ou des travailleurs qui œuvrèrent dans les chantiers navals des détails sur la vie de leurs ancêtres. Toutefois, le choix du titre On chantait "Charley-Man », ainsi que celui de la citation de Narcisse Rosa sur la jaquette arrière, laisse supposer que madame Reid-Marcil nous fera pénétrer au cœur de l'univers social et humain des constructeurs, des ouvriers et de la population québécoise associés à la construction des navires au XIX ${ }^{\mathrm{e}}$ siècle. La citation de Rosa, empreinte d'émotions, parle de sueurs, de bonne humeur, de bruit des vagues et de chants de travail. Il n'en est pourtant rien dans ce présent ouvrage qui s'avère bien plus une description ordonnée qu'un plongeon dans un monde où une certaine charge émotive aurait pu avantageusement être exploitée. L'auteur aurait-il dû englober, pour être fidèle à son titre, les facteurs sociaux qui entourent et définissent la vie des chantiers, le quotidien des marins et celui des passage makers (capitaines qui avaient pour mission de livrer en Angleterre les navires construits à Québec) ou des shipchandlers (accastilleurs de marine) ? - Ces thématiques pourraient probablement faire l'objet d'un autre ouvrage.

ANDré Kirouac

Musée naval de Québec 